\title{
Analysis of Dedifferentiated Liposarcomas Emphasizing the Diagnostic Dilemmas
}

\begin{abstract}
Introduction: Dedifferentiated liposarcoma (DDLPS) is defined as a progression of well-differentiated liposarcoma (WDLPS) to another nonlipogenic sarcoma. Since a variety of heterologous sarcomas can be encountered in dedifferentiation, clinical dilemmas arise. The present study analyzed the role of clinicopathologic and immunohistochemical (IHC) features in the diagnosis of DDLPS and its differentiation from mimics. Materials and Methods: A retrospective and prospective study was conducted wherein all cases of liposarcoma from 2012 to 2017 were reviewed. DDLPS cases were identified among pleomorphic lesions. Clinical and histopathological details for these cases were retrieved from medical records section and department archives. Histomorphology and immunohistochemistry (MDM2, S100, and Ki-67) were analyzed for these cases. Results: Among 37 cases of liposarcomas reviewed, DDLPS was diagnosed in 12 cases (32.4\%). Mean age of the patients was 54.3 years with equal gender distribution $(\mathrm{M}: \mathrm{F}=1: 1.2)$. Two patients had recurrent tumors. Most were retroperitoneal (58.3\%) with mean duration of symptoms being 8.7 months. Mean tumor dimension was $17.5 \mathrm{~cm}$. High-grade dedifferentiated component was most common $(83.3 \%)$ with only one case each $(8.3 \%)$ of low-grade and homologous dedifferentiation. Undifferentiated pleomorphic sarcoma was the frequent nonlipogenic sarcoma. MDM2 overexpression was detected in 100\%, focal S100 positivity seen in 66.6\%, and mean Ki-67 labeling index was 24 . Conclusion: DDLPS exhibits aggressive clinical behavior. Adequate sampling, correlation to clinical details, demonstration of transition from WDLPS to DDLPS aid in narrowing the differentials. Immunostaining with MDM2 helps in definite categorization and S100 highlights lipoblasts, when they are not easily identifiable. MDM2, CDK4, and p16 IHC panel is recommended in all cases and fluorescence in situ hybridization analysis where IHC is noncontributory.
\end{abstract}

Keywords: Dedifferentiated, liposarcoma, MDM2, sarcoma

\section{Introduction}

Liposarcoma is the most common subtype of soft-tissue malignancies, accounting for around $20 \%$ of all adult soft-tissue sarcomas. $^{[1]}$ In the 2013 World Health Organization (WHO) classification, liposarcomas, classified under "adipocytic tumours," are of four major histological subtypes, namely, atypical lipomatous tumor/well-differentiated liposarcoma (ALT/WDLPS), dedifferentiated liposarcoma (DDLPS), myxoid liposarcoma (MLS), and pleomorphic liposarcoma (PLS). ${ }^{[2]}$ These subtypes represent clinical and pathologically distinct entities with discrete cytological and molecular characteristics. $^{[3]}$

The first description and proposal of the term "dedifferentiated" in liposarcoma was

This is an open access journal, and articles are distributed under the terms of the Creative Commons Attribution-NonCommercial-ShareAlike 4.0 License, which allows others to remix, tweak, and build upon the work non-commercially, as long as appropriate credit is given and the new creations are licensed under the identical terms.

For reprints contact: WKHLRPMedknow_reprints@wolterskluwer.com given by Evans in 1979. ${ }^{[4]}$ The progression of an ALT/WDLPS to a sarcoma of variable histological grade (usually nonlipogenic) is regarded as a DDLPS. Around 10\% of ALT/WDLPS show dedifferentiation, the risk being higher in deep-seated and long-standing lesions. ${ }^{[5]}$ Since there can be a wide range of histological morphologies in the dedifferentiated component, they can pose a diagnostic dilemma. This study focuses on the role of clinicopathologic and immunohistochemical (IHC) features in the diagnosis of DDLPS and differentiating it from its mimics. We also discuss in detail the differential diagnosis and approach to the difficult clinical scenarios.

\section{Materials and Methods}

A 5 years' retrospective and prospective study was undertaken in the department of pathology, wherein all cases of liposarcomas

\footnotetext{
How to cite this article: Lali BS, Kini $H$, Chakraborti S, Kini J, Suresh PK. Analysis of dedifferentiated liposarcomas emphasizing the diagnostic dilemmas. Indian J Med Paediatr Oncol 2020;41:39-46.
}

\author{
Bhagat Singh Lali' ${ }^{1}$, \\ Hema Kini', \\ Shrijeet \\ Chakraborti², \\ Jyoti Kini ${ }^{1}$, \\ Pooja K Suresh ${ }^{1}$
}

${ }^{1}$ Department of Pathology, Kasturba Medical College, Manipal Academy of Higher Education, Mangalore, Karnataka, India, ${ }^{2}$ Department of Cellular Pathology, Leighton Hospital, Mid Cheshire NHS Foundation Trust Hospitals, Crewe, England

Submitted: 29-May-2019 Revised: 30-Aug-2019 Accepted: 06-Oct-2019 Published: 24-Apr-2020

Address for correspondence: Dr. Hema Kini, Department of Pathology, Kasturba Medical College, Manipal Academy of Higher Education, Lighthouse Hill Road, Hampankatta, Mangalore - 575 001, Karnataka, India.

E-mail:hema.kini@manipal.edu

Access this article online Website: www.ijmpo.org DOI: 10.4103/ijmpo.ijmpo_129_19 Quick Response Code:

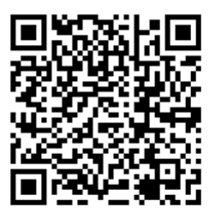




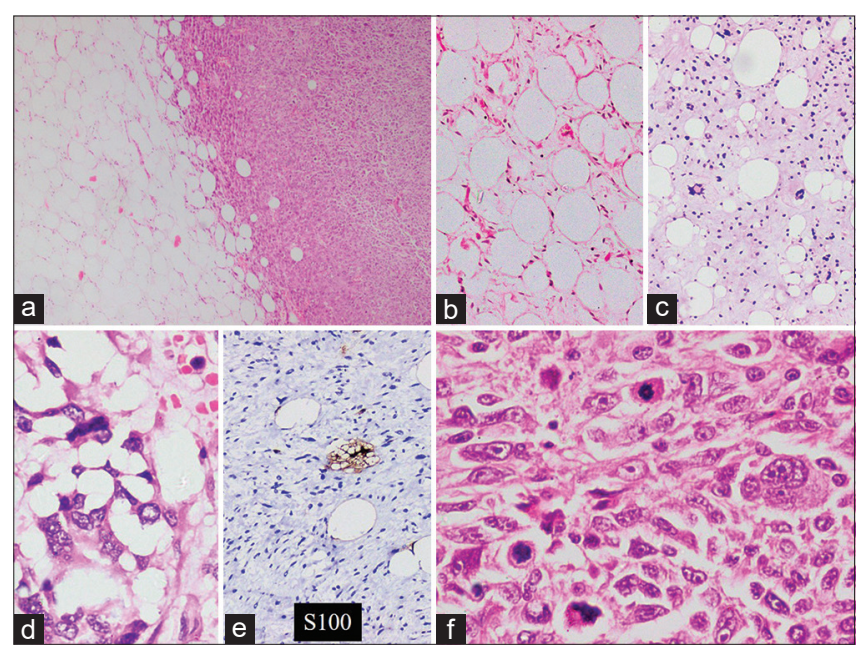

Figure 1: (a) Abrupt transition from well-differentiated liposarcoma to dedifferentiated liposarcoma $(H$ and $E, \times 4)(b)$ Lipoma-like well-differentiated component $(\mathrm{H}$ and $\mathrm{E}, \times 10)(\mathrm{c})$ Sclerosing well-differentiated component $(H$ and $E, \times 10)$ (d and e) Lipoblasts seen within the tumour $(H$ and $E, \times 20)$, highlighted by $\mathrm{S} 100$ immunostain $(\times 20)(f)$ Atypical mitotic figures and tumour giant cells in dedifferentiated component $(H$ and $E, \times 40)$

from January 2012 to December 2017 were reviewed, after attaining the institutional ethics committee approval. The cases with clinical suspicion of liposarcoma, in whom either incisional or excisional biopsy was performed, were included in the study. Cases where tissue or paraffin blocks were not available were excluded.

Details pertaining to the clinical presentation such as duration of symptoms, size and site of the tumor, significant history were collected from the medical records section and departmental archives. Radiology findings, including the computed tomography scans, were also obtained and documented for these cases. The gross examination findings were collected, and microscopy slides were reviewed. Average number of slides reviewed was 17 per case (ranging from 2 to 43). Histologic grade was assigned using the Federation Nationale des Centres de Lutte Contre le Cancer (FNCLCC) grading system. ${ }^{[5]}$ The staging was performed according to the American Joint Committee on Cancer $8^{\text {th }}$ edition. ${ }^{[6]}$

Microscopic details for the WDLPS and DDLPS were individually noted. The dedifferentiation was defined as the presence of homologous or heterologous sarcomatous component of variable histological grade occurring in close proximity to ALT/WDLPS. Areas with increased cellularity, higher mitotic activity, and pleomorphism, as compared to sclerosing WDLPS, were considered as a dedifferentiated component. This must be present in at least one low power field $(\times 10$ objective). The dedifferentiated component is usually required to have a mitotic count of at least 5 mitotic figures per 10 high power fields. ${ }^{[7]}$ Immunostaining with Ki-67 was done for further correlation. The highest grade of the dedifferentiated component was considered for the final impression.

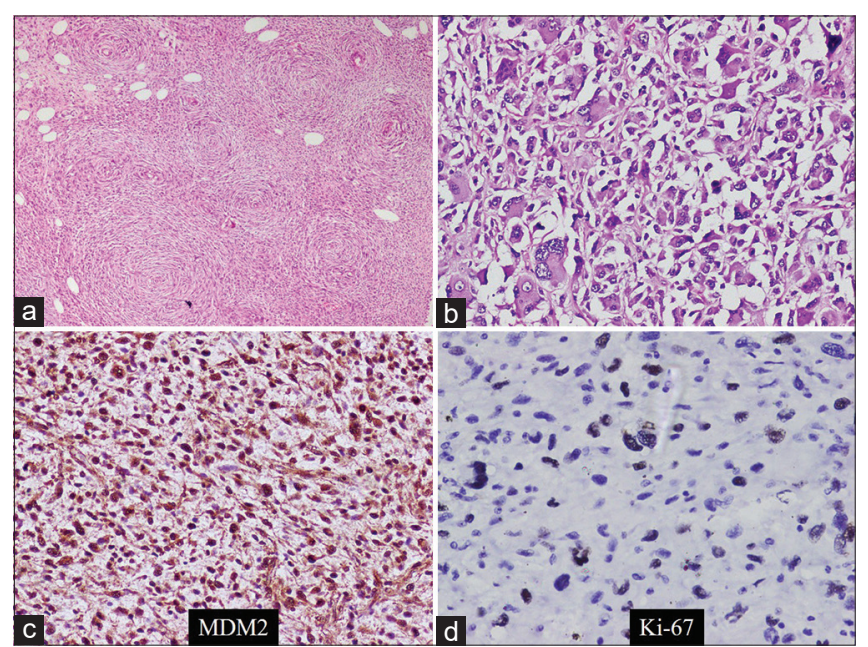

Figure 2: (a) Low-grade component as meningothelial-like whorls (H and $E, \times 4)(b)$ High-grade component as undifferentiated pleomorphic sarcoma $(H$ and $E, \times 20)(c)$ MDM2 immunoreactivity $(\times 20)(d)$ Ki-67 immunostaining $(\times 40)$

IHC staining was performed in all the cases on representative block of formalin-fixed, paraffin-embedded tissue with Murine Double Minute 2 (MDM2) (Abcam; Mouse monoclonal, clone 2A10, Product ID: ab16895), S100 (Dako Flex; Rabbit polyclonal, Ref: IS504), and Ki-67 (Dako Flex; Mouse monoclonal, clone MIB-1, Ref: IS626), according to the instructions provided by the manufacturer. The secondary antibody used was Dako REAL EnVision/ HRP (Labeled polymer, Code K5007) against rabbit and mouse primary antibodies. MDM2 immunostain was read as "positive" when more than $20 \%$ of tumor cells showed moderate-to-strong nuclear positivity. S100 was considered "positive" when nuclear and cytoplasmic staining was seen. Ki-67 labeling index was calculated as the percentage of cells showing nuclear positivity among thousand cells examined at the most mitotically active focus. Additional IHC stains were studied as and when needed in individual cases-desmin, S100, SMA, EMA, bcl-2, CD99, Alk1 to name a few.

The collected data were analyzed with IBM Corp. Released 2015. IBM SPSS Statistics for Windows, Version 23.0. (IBM Corp., Armonk, NY). The percentage analysis was done for categorical data. Mean and standard deviation were studied for continuous variables.

\section{Results}

A total of 45 cases with clinical suspicion of liposarcomas were retrieved. Among them, 37 showed histomorphological features of liposarcomas. Initial diagnosis rendered included DDLPS in eight cases (21.6\%) and PLS in six cases $(16.2 \%)$. After careful microscopic evaluation and immunostaining with MDM2, four PLS cases (10.8\%) were reclassified as DDLPS. Hence, DDLPS was the second-most common subtype, constituting $32.4 \%$ of all LPS. These DDLPS were included in the present study. 
The mean age of the patients was 52.9 years (39-72 years) with majority aged between 60 and 69 years. Almost equal gender distribution ( $\mathrm{M}: \mathrm{F}=1: 1.2)$ was observed. A long-standing mass with a recent increase in size was the most frequent (83.3\%) presenting complaint. Other clinical symptoms included pain, loss of appetite, and weight loss. The mean duration of symptoms was 8.7 months. The most common location was retroperitoneum (58.3\%), followed by trunk and extremities (41.6\%). One case involved the paratesticular region. Two cases had recurrent tumors in the retroperitoneum, previously diagnosed as DDLPS and ALT/ WDLPS. They occurred 3 and 6 years prior to the current presentation, respectively [Table 1].

On radiography, three cases, including the two recurrent tumors, showed extension to the adjacent structures. For instance, one of the recurrent tumors showed the involvement of inferior surface of liver, inferior vena cava, and invasion into anterior abdominal wall. The mean tumor size (greatest dimension) was $17.5 \mathrm{~cm}(4-35 \mathrm{~cm})$. On gross examination, most of the tumors were bosselated, encapsulated masses with yellow to gray-white, necrotic, and hemorrhagic cut surfaces. Myxoid and cystic change was seen focally in a few cases (41.6\%).

Microscopically, in nine cases $(81.8 \%)$, the well-differentiated component was lipoma-like, while two had sclerosing morphology. The transition was abrupt from WDLPS to DDLPS in seven cases (58.3\%) and gradual in four $(33.3 \%)$ [Figure 1a-c]. One case with only an incisional biopsy revealed DDLPS exclusively. The surgical margins were involved in $83.3 \%$ of the tumors, especially those located in the retroperitoneum. The dedifferentiated component was high-grade in eleven cases $(91.6 \%)$ and low-grade in one case $(8.4 \%)$. The high-grade component, in 10 cases, was undifferentiated pleomorphic sarcoma (UPS) showing vague storiform pattern and highly pleomorphic cells with bizarre forms [Figure 2b]. Homologous dedifferentiation from WDLPS to PLS was observed in one case, wherein the latter showed the presence of lipoblasts within the tumor. Low-grade component comprised of meningothelial-like whorls [Figure 2a]. FNCLCC histologic grade was three in most $(83.3 \%)$ of the tumors. On immunohistochemistry, MDM2 was positive in all DDLPS [Figure 2c]. Based on MDM2 positivity, cases which were misclassified as PLS previously were also categorized as DDLPS following review of slides and identification of focal WDLPS areas at the periphery. S100 was focally positive in eight cases $(66.6 \%)$ highlighting the lipoblasts [Figure $1 \mathrm{~d}$ and e]. Mitotic count in all tumors was more than 5/10 high power fields [Figure 1f]. Ki-67 labeling index was variable and ranged from $2 \%$ to $70 \%$ [Figure 2d] with a mean of 24 [Table 1]. Most of the tumors were classified as $\mathrm{pT}_{4}(33.3 \%)$ followed by an equal distribution among $\mathrm{pT}_{3}$ and $\mathrm{pT}_{2}(25 \%$ each). Stage group established in $70 \%$ tumors at presentation was III B. Postoperative radiotherapy was given to $41.6 \%$ of the patients and chemoradiation administered in two cases (16.7\%). On follow-up, one case showed lung and vertebral metastasis after 2 years of the primary diagnosis.

\section{Discussion}

Among all LPS, the initial diagnosis rendered included DDLPS in eight cases $(21.6 \%)$ and PLS in six cases (16.2\%). Four PLS cases (10.8\%) were reclassified as DDLPS on the identification of minimal well-differentiated component and/or MDM2 nuclear positivity. Hence, DDLPS constituted $32.4 \%$ of all LPS. Other authors have reported an incidence of $13.9 \%-16.2 \% .^{[8,9]}$ However, few authors believe that the true incidence of DDLPS is underestimated due to inadequate sampling or misclassification of the lesions. ${ }^{[10]}$ In the recent data at few referral centers, DDLPS is suggested to be the most common histological subtype. ${ }^{[11]}$

The mean age of the patients was 52.9 years with maximum cases presenting in the sixth decade. Almost equal gender distribution ( $\mathrm{M}: \mathrm{F}=1: 1.2)$ was observed. These findings are similar to other studies. ${ }^{[12,13]}$

The most common location of DDLPS in our study was retroperitoneum, and two cases were recurrent tumors. According to the WHO, retroperitoneum is the most common site for DDLPS, outnumbering somatic soft tissue by at least $5: 1 .^{[5]}$ It arises in a recurrent WDLPS or de novo and can itself recur in the retroperitoneal location. ${ }^{[13]}$ The recent increase in the size of a long-standing painless swelling often indicates dedifferentiation, as seen in the present study.

These tumors are usually large in size, especially in the retroperitoneal location. In the present study also, the mean tumor size (greatest dimension) noted was $17.5 \mathrm{~cm}$, with the largest tumor being $35 \mathrm{~cm}$. Microscopically, we found abrupt transition from WDLPS to DDLPS in seven cases $(58.3 \%)$ as was observed by Rekhi et al. in $72 \%$ cases. ${ }^{[13]}$ Margins were involved in $83.3 \%$ of our cases. Margin clearance in retroperitoneal location is challenging due to the extension of the tumor into adjacent structures. Margin positivity adversely affects the prognosis leading to recurrences in these patients. ${ }^{[14]}$

In the present study, the dedifferentiated component encountered frequently was high-grade, in the form of UPS. The low-grade component observed in one case included meningothelial-like whorls. This is in accordance with other authors who also found high-grade dedifferentiation to be more common than low grade. Among high-grade dedifferentiation, they also demonstrated UPS most frequently. ${ }^{[13,15]}$ Homologous dedifferentiation is the terminology proposed by the WHO, in 2013 classification, for tumors showing features of both WDLPS and PLS. ${ }^{[5]}$ Earlier, these were classified as mixed liposarcomas. ${ }^{[16]}$ In our study also, homologous dedifferentiation was found in one case. This can be a potentially misleading point 


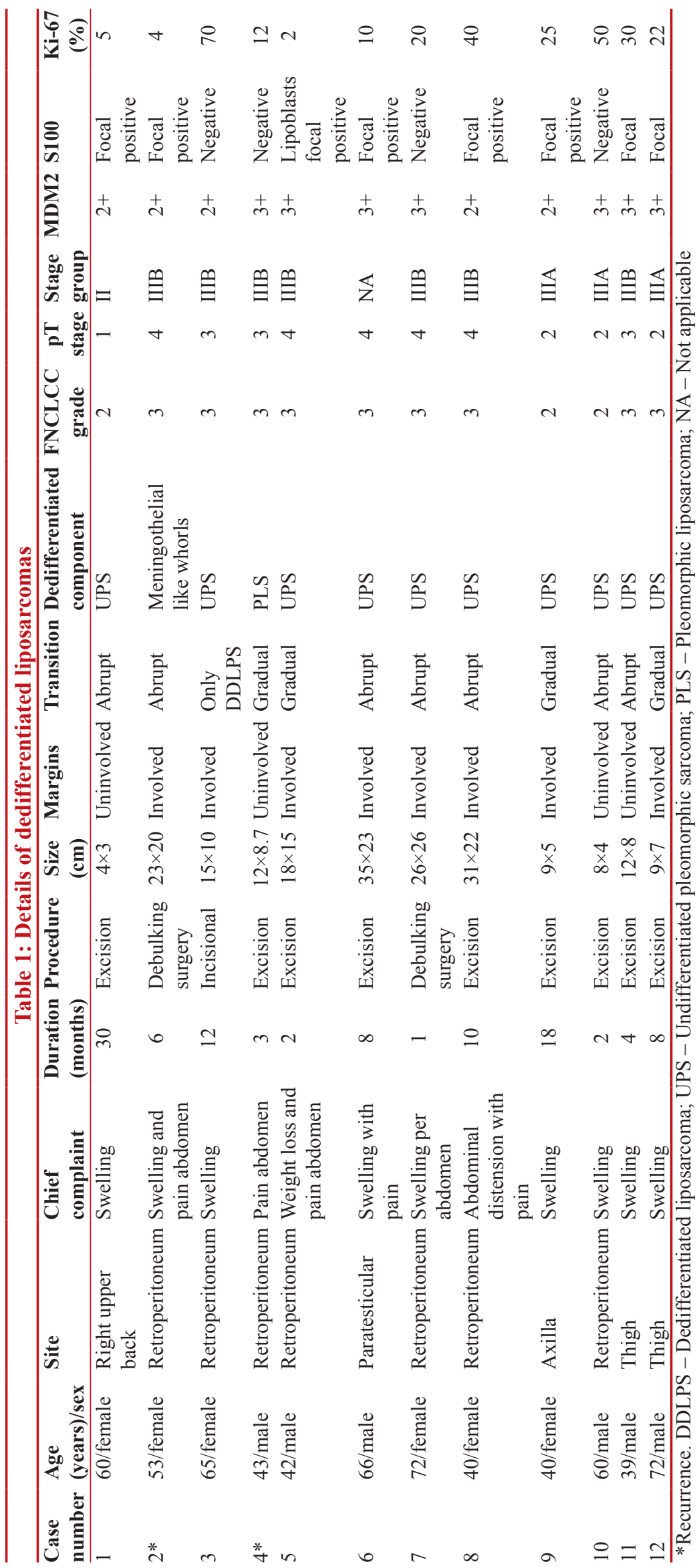


in accurate diagnosis. However, MDM2 positivity in the pleomorphic component facilitates a diagnosis of DDLPS with homologous dedifferentiation. ${ }^{[17]}$

Considering that DDLPS are frequent tumors in the deep-seated soft tissue, especially the retroperitoneum, other high-grade sarcomas are close differentials. The tendency of misdiagnosis is more in the core tissue samples. Ikoma et al., in their study, found the diagnostic sensitivity of percutaneous biopsy to be $37 \%$ in these tumors. The incorrect diagnosis in their study included rhabdomyosarcoma, gastrointestinal stromal tumor (GIST), unclassified sarcoma, malignant fibrous histiocytoma, desmoid tumor, and low-grade B-cell lymphoma. ${ }^{[7]}$

All DDLPS in our study were immunoreactive with MDM2 staining. Kashima et al. found amplification of MDM2 in 90\% DDLPS cases while Touqan et al. reported it in all nine cases $(100 \%) .{ }^{[18,19]} \mathrm{S} 100$ was focally positive in eight cases $(66.6 \%)$, highlighting the lipoblasts in well-differentiated component, in our study. Lipoblasts in the dedifferentiated component were rarely (25\%) positive. Rekhi et al., in their study, found the expression of S-100P in well-differentiated components of six (75\%) DDLPS. It was negative in the dedifferentiated component of seven tumors, except for focal positivity in two tumors containing lipoblasts. ${ }^{[13]} \mathrm{Ki}-67$ labeling index in the present study was ranging from $2 \%$ to $70 \%$ with a mean of 24 , indicative of a wide spectrum of histological grades that can be encountered in these lesions due to tumor heterogeneity.

For MDM2 testing, fluorescence in situ hybridization (FISH) analysis is more sensitive than immunohistochemistry alone. WDLPS and DDLPS harbor ring chromosomes and supernumerary chromosomes and most common mutations from the $12 \mathrm{q} 13-15$ amplicon are MDM2 and CDK4. ${ }^{[5]}$ Thway et al. have reported a high concordance rate of $95.7 \%$ between histology and FISH testing for DDLPS and an overall high concordance rate of 96.5\% between histology and FISH analysis for WDLPS and DDLPS. Moreover, they have discussed that positive results on MDM2 FISH help to exclude the differentials of pleomorphic or MLS, both being close mimickers of DDLPS, in some exceptional circumstances. ${ }^{[20]}$

The consensus for dedifferentiated lipomatous tumors is to use MDM2 and CDK4 immunostaining as a screening procedure, followed by FISH analysis in negative cases. Although the sensitivity of immunohistochemistry is low(around $55 \%-60 \%$ ), the specificity is very high $(90 \%-92 \%)$. Clay et al. concluded that $60 \%$ of patients would require IHC and FISH for a reliable diagnosis. They also opined that cases where slide review and IHC alone are inconclusive constitute only a minority of cases. In resource limited settings like ours, MDM2 and CDK4 can be used for screening followed by ancillary testing recommendation at higher referral centers for cases with high suspicion of DDLPS. On IHC, the addition of p16 to
MDM2 and CDK4 further increases the test sensitivity to $71 \% .^{[21]}$

Differentiating between DDLPS and the invasion of a pleomorphic high-grade tumor into mature fat is a diagnostic dilemma. In such instances, careful observation for the presence of well-differentiated component aids in establishing the diagnosis. Moreover, MDM2 immunostaining is also useful in such cases. ${ }^{[10]}$ Hence, extensive sampling and careful evaluation of the sections for WDLPS is essential. Another diagnostic dilemma arises when an incisional biopsy or core biopsy is performed. If only dedifferentiated part of the tumor is sampled, there is a tendency to misclassify the lesion. Similarly, we had one case wherein incisional biopsy showed features of UPS. The identification of lipoblasts and immunostaining with MDM2 helped in definite categorization. S100 immunostain highlights the lipoblasts, if not readily recognizable.

The differential diagnosis of DDLPS is extensive, and distinction can be challenging at various instances. These tumors can mimic diverse histomorphological patterns seen in tumors ranging from low-grade sarcomas to very pleomorphic high-grade sarcomas. The low-grade pattern can be mistaken for fibromatosis or low-grade fibromyxoid sarcoma. Very inflammatory tumors can mimic sclerosing lesions associated with IgG4 and inflammatory myofibroblastic tumors. Few patterns of DDLPS can even mimic myxoid or PLS subtypes. ${ }^{[2,5,16]}$ A list of differentials with immunohistochemistry and molecular testing approach is shown in Table 2 .

A comprehensive approach is essential for ruling out these differentials. Correlating the site of involvement with peculiar histomorphological features, immunohistochemistry, and molecular genetics helps to rule them out with precision. We discuss here few very close and often misdiagnosed entities.

PLS predominantly occurs in the trunk or extremities of even older population group. Comparatively, they show a greater degree of pleomorphism and more abundant lipoblasts. The presence of lipoblasts is an essential diagnostic criterion in PLS. Homologous dedifferentiation in DDLPS can also pose a challenge in situations where well-differentiated component is minimal or totally absent. PLS is not associated with amplification of MDM2 and/or CDK4 since they are not associated with 12q 13-15 amplicon. ${ }^{[5]}$

MLS also very rarely occur in the retroperitoneum, so much so that it is recommended to aggressively search for a primary elsewhere if MLS is encountered in the retroperitoneum. Moreover, the cellular details are typical for MLS which show small, uniform, ovoid nuclei with bland morphology. On the contrary, myxoid areas in DDLPS still show at least focal nuclear atypia and pleomorphism. MLS harbor DDIT3-FUS fusion mutations and rarely, DDIT3-EWSR1 fusion. ${ }^{[2,5]}$ 


\begin{tabular}{|c|c|c|c|}
\hline Differential diagnosis & DDLPS $^{[2,3]}$ & Immunohistochemistry $^{[5,13,22]}$ & Molecular findings ${ }^{[5,13,22]}$ \\
\hline \multicolumn{4}{|c|}{ DDLPS with low-grade dedifferentiation } \\
\hline Fibromatosis & $\begin{array}{l}\text { MDM2 (IHC and/or FISH), } \\
\text { CDK4, p16 }\end{array}$ & $\begin{array}{l}\text { Beta catenin, Cyclin D1, } \\
\text { Calretinin }\end{array}$ & CTNNB1 \\
\hline $\begin{array}{l}\text { Low grade fibromyxoid } \\
\text { sarcoma }\end{array}$ & & MUC4, CD99, Bcl2 & t $(7 ; 16)$ with FUS-CREB3L2 fusion \\
\hline $\begin{array}{l}\text { Inflammatory myofibroblastic } \\
\text { tumor }\end{array}$ & & $\begin{array}{l}\text { Vimentin, alpha smooth muscle } \\
\text { actin, muscle specific actin, Alk1 }\end{array}$ & $\begin{array}{l}\mathrm{t}(2 ; 5)-\text { ALK and NPM, } \\
\mathrm{t}(2 ; 17)-\text { ALK and CLTC }\end{array}$ \\
\hline \multicolumn{4}{|c|}{ DDLPS with high-grade dedifferentiation } \\
\hline High-grade fibrosarcoma & MDM2 (IHC and/or FISH), & Vimentin, p53, type 1 collagen & Aneuploid \\
\hline SFT with dedifferentiation & $\mathrm{CDK} 4, \mathrm{p} 16$ & CD34, CD99, STAT6 & Non-specific \\
\hline $\begin{array}{l}\text { Malignant peripheral nerve } \\
\text { sheath tumor }\end{array}$ & & $\begin{array}{l}\text { CD99, S100, Leu7/CD57 } \\
\text { (in neurofibroma-like areas) }\end{array}$ & Non-specific \\
\hline Synovial sarcoma & & TLE1, Cytokeratins, Bcl2, CD99 & $\begin{array}{l}\mathrm{t}(\mathrm{X} ; 18)-\mathrm{SYT}-\mathrm{SSX} 1 \text { or } \\
\text { SYT-SSX2 }\end{array}$ \\
\hline High-grade myxofibrosarcoma & & No specific stains, CD34 & $\begin{array}{l}\text { Highly complex karyotypes, often } \\
6 \mathrm{p}-, 9 \mathrm{q}+, 12 \mathrm{q}+\end{array}$ \\
\hline $\begin{array}{l}\text { Spindle cell } \\
\text { rhabdomyosarcoma }\end{array}$ & & $\begin{array}{l}\text { MyoD1 (diffuse), Desmin, } \\
\text { Myogenin, Actin (focal) }\end{array}$ & Recurrent MyoD1 mutations \\
\hline Pleomorphic liposarcoma & & Vimentin, S100, SMA & Complex karyotypes \\
\hline $\begin{array}{l}\text { Undifferentiated pleomorphic } \\
\text { sarcoma }\end{array}$ & & $\begin{array}{l}\text { Diagnosis of exclusion, focal } \\
\text { SMA positivity (few cases) }\end{array}$ & Complex karyotypes \\
\hline
\end{tabular}

Morphologically, IgG4 disease exhibits dense inflammatory infiltrate with storiform fibrosis. However, IgG4 related sclerosing lesions can easily be ruled out by correlation with systemic features since IgG4 related disease is now recognized as systemic disorder, characterized by high serum IgG4 levels (exceeding $135 \mathrm{mg} / \mathrm{dl}$ ) and more than 10 IgG4 positive cells per high-power field. ${ }^{[22]}$ Inflammatory myofibroblastic tumor involves the retroperitoneum quite often, especially in children and young adults. On morphology, it typically displays a mixture of three patterns- nodular fasciitis like cellular areas, spindled myofibroblasts and densely hyalinized stroma. However, unlike the inflammatory DDLPS, all these patterns do not display pleomorphism or atypical mitotic figures. IHC with alpha-smooth muscle actin, calponin, and Alk1 can help in the distinction in conjunction with MDM2 and CDK4. ${ }^{[23]}$

Solitary fibrous tumor (SFT) can also arise in the retroperitoneum and can rarely dedifferentiate. SFT harbors NAB2-STAT6 fusion transcripts with nuclear expression of STAT6 protein. A subset of DDLPS has also been found to express STAT6 and CD34. In such situations, diffuse expression of CD34, CD99, and bcl-2 can help to give a final call of SFT. All three are very unlikely to be expressed diffusely in DDLPS. ${ }^{[5,23]}$

Synovial sarcomas can involve the intra-abdominal sites, and though they are composed of monotonous population of cells, the spindle cells may show some degree of pleomorphism. However, the focal expression of cytokeratins, epithelial membrane antigen, and TLE1 positivity along with specific translocation $t(\mathrm{X} ; 18)(\mathrm{p} 11.2$; q11.2) saves the day often.

Malignant peripheral nerve sheath tumors (MPNST) can occur in the abdomen, show varied morphology with dedifferentiation to any lineage. Hence, the distinction can be challenging. Morphologically, MPNST will show, at least focally, cells with elongated, tapered hyperchromatic nuclei suggesting the neural origin. Moreover, they tend to lack the expression of MDM2, p16 or CDK4 and express CD99, S100 or p53. ${ }^{[24]}$

DDLPS can rarely show immunopositivity for CD117 and misdiagnosed as retroperitoneal GIST. GIST displays more uniform spindle cells lacking atypia. GIST expresses CD117 more diffusely and is typically positive for DOG-1. Moreover, they harbor KIT or PDGFRA mutations. ${ }^{[23]}$

One $(8.3 \%)$ of our patients presented with pulmonary and vertebral metastasis after 2 years of primary diagnosis. Distant metastasis is usually observed in $15 \%-20 \%$ of DDLPS, tumors metastasizing frequently to lungs. ${ }^{[5]}$ One case $(8.3 \%)$ had recurrent DDLPS after 3 years in our study. The local recurrence rate is very high in DDLPS varying from $41 \%$ to $50 \%$ in various studies. ${ }^{[8,12]}$ The recurrence duration in these studies is documented to be within a time span of 8.5 months -60 months. ${ }^{[8,12,25]}$ Oh et al. found the 5 -year disease-free survival of $22.1 \%$ and overall 5-year survival of $49 \%$ in DDLPS patients. ${ }^{[9]}$

Treatment options available for DDLPS include surgical resection, often associated with radiotherapy and/or 
chemotherapy. MDM2 not only aids in the diagnosis of liposarcomas but also has been targeted by drugs, in both WDLPS and DDLPS. Nutlin is the first specific and potent MDM2 inhibitor discovered. Research is ongoing for other MDM2 inhibitors, including AT-219, RG7388, and Ascenta. ${ }^{[26]}$ Considering the aggressive biological behavior of DDLPS, potential of targeted therapy and low disease-free survival rates, correct identification of these lesions constitutes an important milestone in patient management.

The limitation of the present study is the standalone use of MDM2 immunohistochemistry without FISH analysis, due to nonavailability at the center. A larger study with a greater number of cases and FISH analyses can help create an approach to the diagnosis of this category of tumors.

\section{Conclusion}

Dedifferentiated LPS is a subtype of liposarcomas with more aggressive clinical behavior. They pose diagnostic dilemmas in unusual case scenarios. Adequate sampling of the lesion, correlation with clinical details, and demonstration of the transition of WDLPS to DDLPS aid in narrowing down the differentials. Immunostaining with MDM2 can help in definite categorization with high specificity. S100 staining can highlight the lipoblasts in tumors where they are not easily identifiable. A panel of IHC recommended for DDLPS must include MDM2, CDK4, and p16. Analysis with MDM2 FISH amplification is essential for cases where IHC is noncontributory.

\section{Financial support and sponsorship}

Nil.

\section{Conflicts of interest}

There are no conflicts of interest.

\section{References}

1. Brennan MF, Antonescu CR, Moraco N, Singer S. Lessons learned from the study of 10,000 patients with soft tissue sarcoma. Ann Surg 2014;260:416-21.

2. Jo VY, Fletcher CD. WHO classification of soft tissue tumours: An update based on the $2013\left(4^{\text {th }}\right)$ edition. Pathology 2014;46:95-104.

3. de Vreeze RS, de Jong D, Nederlof PM, Ariaens A, Tielen IH, Frenken L, et al. Added value of molecular biological analysis in diagnosis and clinical management of liposarcoma: A 30-year single-institution experience. Ann Surg Oncol 2010;17:686-93.

4. Evans HL. Liposarcoma: A study of 55 cases with a reassessment of its classification. Am J Surg Pathol 1979;3:507-23.

5. Fletcher CD, Bridge JA, Hogendoorn PC, Mertens F. Adipocytic tumours. In: WHO Classification of Tumours of Soft Tissue and Bone. $4^{\text {th }}$ ed. Lyon: International Agency for Research on Cancer; 2013. p. 33-43.

6. Amin MB. AJCC Cancer Staging Manual. $8^{\text {th }}$ ed. Chicago: Springer US; 2017. p. 489-548.
7. Ikoma N, Torres KE, Somaiah N, Hunt KK, Cormier JN, Tseng W, et al. Accuracy of preoperative percutaneous biopsy for the diagnosis of retroperitoneal liposarcoma subtypes. Ann Surg Oncol 2015;22:1068-72.

8. Knebel C, Lenze U, Pohlig F, Lenze F, Harrasser N, Suren $\mathrm{C}$, et al. Prognostic factors and outcome of liposarcoma patients: A retrospective evaluation over 15 years. BMC Cancer 2017; 17:410.

9. Oh YJ, Yi SY, Kim KH, Cho YJ, Beum SH, Lee YH, et al. Prognostic model to predict survival outcome for curatively resected liposarcoma: A multi-institutional experience. J Cancer 2016;7:1174-80.

10. Dei Tos AP. Liposarcomas: Diagnostic pitfalls and new insights. Histopathology 2014;64:38-52.

11. Mastrangelo G, Coindre JM, Ducimetière F, Dei Tos AP, Fadda E, Blay JY, et al. Incidence of soft tissue sarcoma and beyond: A population-based prospective study in 3 European regions. Cancer 2012;118:5339-48.

12. Kalimuthu SN, Tilley C, Forbes G, Ye H, Lehovsky K, Pillay N, et al. Clinical outcome in patients with peripherally-sited atypical lipomatous tumours and dedifferentiated liposarcoma. J Pathol Clin Res 2015;1:106-12.

13. Rekhi B, Navale P, Jambhekar NA. Critical histopathological analysis of 25 dedifferentiated liposarcomas, including uncommon variants, reviewed at a tertiary cancer referral center. Indian J Pathol Microbiol 2012;55:294-302.

14. Singer S, Antonescu CR, Riedel E, Brennan MF. Histologic subtype and margin of resection predict pattern of recurrence and survival for retroperitoneal liposarcoma. Ann Surg 2003;238:358-70.

15. Lu W, Lau J, Xu MD, Zhang Y, Jiang Y, Tong HX, et al. Recurrent abdominal liposarcoma: Analysis of 19 cases and prognostic factors. World J Gastroenterol 2013;19:4045-52.

16. Fletcher CD, Unni KK, Mertens F. Adipocytic tumours. In: Pathology and Genetics of Tumours of Soft Tissue and Bone. $3^{\text {rd }}$ ed. Lyon: International Agency for Research on Cancer; 2002. p. 35-46.

17. Mariño-Enríquez A, Fletcher CD, Dal Cin P, Hornick JL. Dedifferentiated liposarcoma with "homologous" lipoblastic (pleomorphic liposarcoma-like) differentiation: Clinicopathologic and molecular analysis of a series suggesting revised diagnostic criteria. Am J Surg Pathol 2010;34:1122-31.

18. Kashima T, Halai D, Ye H, Hing SN, Delaney D, Pollock R, et al. Sensitivity of MDM2 amplification and unexpected multiple faint alphoid 12 (alpha 12 satellite sequences) signals in atypical lipomatous tumor. Mod Pathol 2012;25:1384-96

19. Touqan N, Diggle CP, Verghese ET, Perry S, Horgan K, Merchant $\mathrm{W}$, et al. An observational study on the expression levels of MDM2 and MDMX proteins, and associated effects on P53 in a series of human liposarcomas. BMC Clin Pathol 2013;13:32.

20. Thway K, Wang J, Swansbury J, Min T, Fisher C. Fluorescence in situ hybridization for MDM2 amplification as a routine ancillary diagnostic tool for suspected well-differentiated and dedifferentiated liposarcomas: Experience at a tertiary center. Sarcoma 2015;2015:1-10.

21. Clay MR, Martinez AP, Weiss SW, Edgar MA. MDM2 and CDK4 immunohistochemistry: Should it be used in problematic differentiated lipomatous tumors?: A new perspective. Am J Surg Pathol 2016;40:1647-52.

22. Umehara H, Okazaki K, Masaki Y, Kawano M, Yamamoto M, 
Saeki T, et al. Comprehensive diagnostic criteria for IgG4-related disease (IgG4-RD), 2011. Mod Rheumatol 2012;22:21-30.

23. Thway K, Jones RL, Noujaim J, Zaidi S, Miah AB, Fisher C. Dedifferentiated liposarcoma: Updates on morphology, genetics, and therapeutic strategies. Adv Anat Pathol 2016;23:30-40.

24. Fletcher CD. Tumors of soft tissue. In: Diagnostic Histopathology of Tumors. $4^{\text {th }}$ ed. Philadelphia: Elsevier; 2013. p. 1804-9.
25. Chen C, Borker R, Ewing J, Tseng WY, Hackshaw MD, Saravanan $\mathrm{S}$, et al. Epidemiology, treatment patterns, and outcomes of metastatic soft tissue sarcoma in a community-based oncology network. Sarcoma 2014;2014:1-7.

26. Patel RB, Li T, Liao Z, Jaldeepbhai JA, Perera HA, Muthukuda SK, et al. Recent translational research into targeted therapy for liposarcoma. Stem Cell Investig 2017;4:21. 